Research Article

\title{
Important Issues on Spectral Properties of a Transmission Eigenvalue Problem
}

\author{
Besiana Cobani $\mathbb{D}^{1},{ }^{1}$ Aurora Simoni $\mathbb{D}^{2},{ }^{2}$ and Ledia Subashi $\mathbb{D}^{1}$ \\ ${ }^{1}$ Department of Mathematics, Faculty of Natural Science, University of Tirana, Tirana, Albania \\ ${ }^{2}$ Department of Applied Mathematics, Faculty of Natural Science, University of Tirana, Tirana, Albania \\ Correspondence should be addressed to Besiana Cobani; besiana.hamzallari@fshn.edu.al
}

Received 17 June 2021; Accepted 18 August 2021; Published 31 August 2021

Academic Editor: Jaume Giné

Copyright (c) 2021 Besiana Cobani et al. This is an open access article distributed under the Creative Commons Attribution License, which permits unrestricted use, distribution, and reproduction in any medium, provided the original work is properly cited.

\begin{abstract}
Nowadays, inverse scattering is an important field of interest for many mathematicians who deal with partial differential equations theory, and the research in inverse scattering is in continuous progress. There are many problems related to scattering by an inhomogeneous media. Here, we study the transmission eigenvalue problem corresponding to a new scattering problem, where boundary conditions differ from any other interior problem studied previously. more specifically, instead of prescribing the difference Cauchy data on the boundary which is the classical form of the problem, we consider the case when the difference of the trace of the fields is proportional to the normal derivative of the field. Typical concerns related to TEP (transmission eigenvalue problem) are Fredholm property and solvability, the discreteness of the transmission eigenvalues, and their existence. In this article, we provide answers for all these concerns in a given interior transmission problem for an inhomogeneous media. We use the variational method and a very important theorem on the existence of transmission eigenvalues to arrive at the conclusion of the existence of the transmission eigenvalues.
\end{abstract}

\section{Introduction}

To study thoroughly a problem in inverse scattering for an inhomogeneous media with a conductive boundary, we should deal with its interior transmission problem. Its homogeneous version is referred to as the transmission eigenvalue problem. The TEP (transmission eigenvalue problem) is nonlinear and not self-adjoint $[1,2]$. In this paper, the focus is to prove the discreteness and the existence of real transmission eigenvalues of a given problem.

The discreteness of transmission eigenvalues is important to prove the solvability of the interior transmission problem because the latter satisfies the Fredholm Alternative. From a practical point of view the discreteness is needed to guarantee that the reconstruction methods, such as linear sampling, succeed in the reconstruction of the scatterer of an inhomogeneous medium $[3,4]$. The importance of the existence of transmission eigenvalues is that they determinate data about the material properties of the inhomogeneous media. Besides the theoretical importance of transmission eigenvalues in connection with uniqueness and reconstruction results in inverse scattering theory, recently they have been used to obtain information about the index of refraction from measured data [5]. This is based on the important result that transmission eigenvalues can be determined from the measured far field data $[2,4]$. The interior problem is the key problem in inverse scattering. After studying thoroughly the issue of the well-posedness of the direct problem, [6] which is necessary to continue with the inverse problem, we focus our work in the solution of the interior problem which is the central problem in inverse scattering. In inhomogeneous media, the issue of solving the interior problem is related to the transmission eigenvalues. The question that concerns the most is the existence of transmission eigenvalues [4].

In this paper, we study the discreteness and the existence of real transmission eigenvalues associated with the following scattering problem: let $\Omega \subset \mathbb{R}^{d}, d \in\{2,3\}$ be a 
collection of bounded simply connected domains with piecewise smooth boundary $\partial \Omega$, and with connected exterior $\mathbb{R}^{d} / \bar{\Omega}$, and let the bounded and real-valued function $n$ denote the refractive index, $v$ the outward unit normal to the boundary $\partial \Omega, k>0$ the wave number, and $\eta$ a real-valued, positive boundary parameter [7]. We assume that $\Omega$ is given. The interior transmission eigenvalue problem corresponding to the scattering problem is to determine the values $k \in \mathbb{C}$ such that there exists a nontrivial solution to

$$
\begin{aligned}
\Delta w+k^{2} n w & =0, \quad \text { in } \Omega, \\
\Delta v+k^{2} v & =0, \quad \text { in } \Omega, \\
w-v & =-\eta \frac{\partial v}{\partial \nu}, \quad \text { on } \partial \Omega, \\
\frac{\partial w}{\partial \nu}-\frac{\partial v}{\partial v} & =0, \quad \text { on } \partial \Omega .
\end{aligned}
$$

We call such values of $k$ the interior transmission eigenvalues. This problem is known to be non-self-adjoint eigenvalue problem [8]. The goal is to show that the set of transmission eigenvalues (real and complex) is at most discrete and then prove the existence of real transmission eigenvalues.

To this end, we first formulate the respective problem in the variational form $[3,9]$. For this propose, we operate in the Sobolev space

$$
\tilde{H}_{0}^{2}(\Omega)=\left\{u \in H^{2}(\Omega), \quad \text { such that } \frac{\partial u}{\partial \nu}=0 \text {, on } \partial \Omega\right\} \text {. }
$$

The space $\tilde{H}_{0}^{2}(\Omega)$ is equipped with $\|\cdot\|_{H^{2}(\Omega)}$ norm [2]. Then, we give a more formal presentation of the interior problem as a quadratic form related to $k$, where all the operators present are compact and self-adjoint. From spectral theory, the discreetness follows $[2,10]$. The most important part of the study is showing the existence of real transmission eigenvalues. To this end, we rely on the variational formulation $[9,11]$ of the transmission eigenvalue problem, and we use a very important theorem of [8].

\section{The Discreteness of the Transmission Eigenvalues}

For analytic consideration, we make the following assumptions [2]: the boundary $\partial \Omega$ is of class $C^{2}, n \in L^{\infty}(\Omega)$ is real-valued and there exists $\alpha$ such that $0<n_{*}<1$ or $n^{*}>1$, where

$$
\begin{aligned}
& n_{*}=\inf _{x \in \Omega} n(x), \\
& n^{*}=\sup _{x \in \Omega} n(x) .
\end{aligned}
$$

Furthermore, $\eta \in L^{\infty}(\partial \Omega)$ is real-valued such that $\eta>0$.

The transmission eigenvalue problem is as follows: for given functions $n \in L^{\infty}(\Omega)$ and $\eta \in L^{\infty}(\partial \Omega)$ find $k>0$ and $(w, v) \in L^{2}(\Omega) \times L^{2}(\Omega) \quad$ such that $(\partial w / \partial v)-(\partial v / \partial v) \in \widetilde{H}_{0}^{2}(\Omega)$ and $(w, v)$ satisfies (1)-(4).

Next, we reformulate this transmission eigenvalue problem as an eigenvalue problem for a fourth-order differential operator. To this end, let $u \in \widetilde{H}_{0}^{2}(\Omega)$ denote the difference $w-v$. Then, $u$ satisfies

$$
\left(\Delta+k^{2} n\right) u=-k^{2}(n-1) v, \quad \text { in } \Omega .
$$

Applying the operator $\left(\Delta+k^{2}\right)$ to both sides, we have

$$
\left(\Delta+k^{2}\right) \frac{1}{n-1}\left(\Delta+k^{2} n\right) u=0, \quad \text { in } \Omega .
$$

Note that $n-1$ is either strictly positive or strictly negative in $\Omega$ thanks to the above assumptions on $n$. From the relation (5), the function $v$ can be given as $v=\left(1 /-k^{2}(n-1)\right)\left(\Delta+k^{2} n\right) u$, and as a result, we write the boundary condition (3) as

$$
u=\frac{\eta}{k^{2}} \frac{\partial}{\partial v} \frac{1}{n-1}\left(\Delta+k^{2} n\right) u, \quad \text { on } \partial \Omega .
$$

Multiplying with a test function $\bar{\varphi}$, identity (8), and integrating over $\Omega$, we obtain

$$
\int_{\Omega}\left(\Delta+k^{2}\right) \frac{1}{n-1}\left(\Delta+k^{2} n u\right) \bar{\varphi} \mathrm{d} x=0, \quad \forall \varphi \in \tilde{H}_{0}^{2}(\Omega),
$$

and using the boundary condition (7) and Green's identity, we arrive at

$$
\int_{\partial \Omega} \bar{\varphi} \frac{k^{2} u}{\eta} \mathrm{d} s-\int_{\Omega} \frac{1}{n-1}\left(\Delta u+k^{2} n u\right) \cdot\left(\Delta \bar{\varphi}+k^{2} \bar{\varphi}\right) \mathrm{d} x=0
$$

Hence, the transmission eigenvalue problem becomes a quadratic form related to $k$. This is important to prove the discreteness of transmission eigenvalues [2].

We operate on the variational (11) rewritten as

$$
\int_{\Omega} \frac{1}{n-1}\left(\Delta u+k^{2} n u\right) \cdot\left(\Delta \bar{\varphi}+k^{2} \bar{\varphi}\right) \mathrm{d} x-\int_{\partial \Omega} \bar{\varphi} \frac{k^{2} u}{\eta} \mathrm{d} s=0, \quad \forall \varphi \in \widetilde{H}_{0}^{2}(\Omega),
$$

where we group each factor in terms of $k$ as follows:

$$
\int_{\Omega} \frac{1}{n-1} \Delta u \cdot \Delta \bar{\varphi} \mathrm{d} x-k^{2} \int_{\partial \Omega} \bar{\varphi} \frac{u}{\eta} \mathrm{d} s+k^{2} \int_{\Omega} \frac{1}{n-1}(\Delta u \bar{\varphi}+n u \Delta \bar{\varphi}) \mathrm{d} x+k^{4} \int_{\Omega} \frac{1}{n-1} n u \cdot \bar{\varphi} \mathrm{d} x=0 .
$$


By the Riesz representation theorem $[9,11,12]$, we by define the following operators:

$$
T: \widetilde{H}_{0}^{2}(\Omega) \longrightarrow \widetilde{H}_{0}^{2}(\Omega),
$$

such that

$$
\begin{aligned}
(T u, \varphi)= & \int_{\Omega} \frac{1}{n-1} \Delta u \cdot \Delta \bar{\varphi} \mathrm{d} x+\int_{\Omega} \nabla u \cdot \nabla \bar{\varphi} \mathrm{d} x+\int_{\Omega} u \cdot \bar{\varphi} \mathrm{d} x, \\
& T_{k}: \widetilde{H}_{0}^{2}(\Omega) \longrightarrow \widetilde{H}_{0}^{2}(\Omega),
\end{aligned}
$$

$$
\left(T_{k} u, \varphi\right)=-\int_{\Omega} \nabla u \cdot \nabla \bar{\varphi} d x-\int_{\Omega} u \cdot \bar{\varphi} d x-k^{2} \int_{\partial \Omega} \bar{\varphi} \frac{u}{\eta} \mathrm{d} s+k^{2} \int_{\Omega} \frac{1}{n-1}(\Delta u \bar{\varphi}+n u \Delta \bar{\varphi}) \mathrm{d} x+k^{4} \int_{\Omega} \frac{1}{n-1} n u \cdot \bar{\varphi} \mathrm{d} x
$$

Then, the problem can be written as

$$
T u+T_{k} u=0, \quad \forall \varphi \in \widetilde{H}_{0}^{2}(\Omega) .
$$

Next, we prove that the operator $T$ is invertible, and the operator $T^{-1}: \widetilde{H}_{0}^{2}(\Omega) \longrightarrow \widetilde{H}_{0}^{2}(\Omega)$ is bounded. This assertion follows from the coercivity of the following sesquilinear form:

$$
a(\cdot, \cdot):=\int_{\Omega} \frac{1}{n-1} \Delta u \cdot \Delta \bar{\varphi} \mathrm{d} x+\int_{\Omega} \nabla u \cdot \nabla \bar{\varphi} \mathrm{d} x+\int_{\Omega} u \cdot \bar{\varphi} \mathrm{d} x
$$

is coercive, that is proven by the following inequalities:

$$
\begin{aligned}
|a(u, \varphi)| \geq & \frac{1}{n_{*}-1}\|\Delta u\|_{L^{2}(\Omega)}\|\Delta \varphi\|_{L^{2}(\Omega)}+\|\nabla u\|_{L^{2}(\Omega)}\|\nabla \varphi\|_{L^{2}(\Omega)} \\
& +\|u\|_{L^{2}(\Omega)}\|\varphi\|_{L^{2}(\Omega)} \\
\geq & c\|\nabla u\|_{H^{2}(\Omega)}\|\nabla \varphi\|_{H^{2}(\Omega)} .
\end{aligned}
$$

Theorem 1. The operator $T_{K}: \tilde{H}_{0}^{2}(\Omega) \longrightarrow \tilde{H}_{0}^{2}(\Omega)$ is compact.

Proof. Let $u \in H_{0}^{2}(\Omega)$ which is compactly embedded in $H^{2}(\Omega)$.

$$
\begin{aligned}
\nabla u & \in H^{1}(\Omega), \\
u & \in H^{2}(\Omega), \\
\left.u\right|_{\partial \Omega} & \in H^{3 / 2} \partial \Omega .
\end{aligned}
$$

Thus, $\int_{\Omega} \nabla u \cdot \nabla \bar{\varphi} \mathrm{d} x$ contains $L^{2}(\Omega)$ norm of $\nabla u$ which is in $H^{1}(\Omega)$ and $H^{1}(\Omega)$ is compactly embedded in $L^{2}(\Omega)$. Furthermore, $\int_{\Omega} \nabla u \cdot \nabla \bar{\varphi} \mathrm{d} x$ contains $L^{2}(\Omega)$ norm of $u$ and $H^{2}(\Omega)$ is compactly embedded in $L^{2}(\Omega)$. For the third integral, $\int_{\partial \Omega}(1 / n) u \cdot \bar{\varphi}$ contains $L^{2}(\partial \Omega)$ norm of $u$ because $u$ is in $H^{3 / 2}(\partial \Omega)$ which is compactly embedded in $L^{2}(\partial \Omega)$. We have the following relations using Green's first identity:

$$
\int_{\Omega} \frac{1}{n-1} \Delta u \cdot \bar{\varphi} \mathrm{d} x=-\int_{\Omega} \nabla u \cdot \nabla \frac{1}{n-1} \bar{\varphi} \mathrm{d} x+\int_{\partial \Omega} \frac{\partial u}{\partial \nu} \cdot \frac{1}{n-1} \bar{\varphi} \mathrm{d} s=-\int_{\Omega} \nabla u \cdot \nabla \frac{1}{n-1} \bar{\varphi} \mathrm{d} x
$$

Hence,

$$
\int_{\Omega} \frac{1}{n-1} \Delta u \cdot \bar{\varphi} \mathrm{d} x=-\int_{\Omega} \nabla u \cdot \nabla \frac{1}{n-1} \bar{\varphi} \mathrm{d} x
$$

and $\nabla u$ is in $H^{1}(\Omega)$. Thus, the same as above $H^{1}(\Omega)$ is compactly embedded in $L^{2}(\Omega)$.

Finally, from the above, we can write (17)

$$
\left(I+T^{-1} T_{k}\right) u=0, \quad \forall u \in H_{0}^{2}(\Omega) .
$$

The operator $T_{k}$ is an analytic operator valued (it is polynomial in $k \in \mathbb{C}$ ). The analytic Fredholm theory implies that

$$
\left(I+T^{-1} T_{k}\right)
$$

has an empty kernel for all $k \in \mathbb{C}$, except for possibly a discrete set, if we find one $k$ for which the kernel is empty. Now, in Section 3, we show that $k$ real and small enough are not transmission eigenvalues. Hence, the set of transmission eigenvalues in the complex plane is discrete with $+\infty$ as the only accumulation point. 


\section{The Existence of Real Transmission Eigenvalues}

The study of existence of transmission eigenvalues is based in the following theorem (Cakoni-Haddar) [8].

Theorem 2. Let $\tau \longrightarrow A_{\tau}$ be a continuous mapping from ] $0, \infty$ [ to the set of self-adjoint and positive definite bounded linear operators on $U$, where $U$ is a separable Hilbert space, and let $B$ be a self-adjoint and nonnegative compact bounded linear operator on $U$. We assume that there exist two positive constants $\tau_{0}>0$ and $\tau_{1}>0$ such that

(1) $A_{\tau_{0}}-\tau_{0} B$ is positive on $U$.

(2) $A_{\tau 1}-\tau_{1} B$ is non-positive on a $k$ dimensional subspace $W_{k}$ of $U$. Then, each of the equations $\lambda_{j}(\tau)=\tau$ for $j=\overline{1, k}$ has at least one solution in $\left[\tau_{0}, \tau_{1}\right]$ where $\lambda_{j}(\tau)$ is the $j$ th eigenvalue (counting multiplicity) of $A_{\tau}$ with respect to $B$.

To use the above theorem, we have to write relation (11) as $A_{\tau}-\tau B=0$, where $A_{\tau}$ is a family of positive definite selfadjoint bounded linear operator, $B$ is a nonnegative compact bounded linear operator, defined on appropriate Hilbert spaces. Then, a transmission eigenvalue is the solution of $\lambda(\tau)-\tau=0$, where $\lambda(\tau)$ is an eigenvalue of the generalized eigenvalue problem $A_{\tau}-\tau B=0$, where in our case $\tau:=k^{2}$ [13]. In this section, we assume that $k$ is real.

Let us first recall the variational formulation (11):

$$
\int_{\partial \Omega} \bar{\varphi} \frac{k^{2} u}{\eta} \mathrm{d} s-\int_{\Omega} \frac{1}{n-1}\left(\Delta u+k^{2} n u\right) \cdot\left(\Delta \bar{\varphi}+k^{2} \bar{\varphi}\right) \mathrm{d} x=0, \quad \forall \varphi \in \widetilde{H}_{0}^{2}(\Omega) .
$$

To have the generalized eigenvalue problem $A_{\tau}-\tau B=0$, we do the following operation in (11):

$$
\begin{aligned}
& \int_{\partial \Omega} \bar{\varphi} \frac{k^{2} u}{\eta} \mathrm{d} s-\int_{\Omega} \frac{1}{n-1}\left(\Delta u+k^{2}(n-1+1) u\right) \cdot\left(\Delta \bar{\varphi}+k^{2} \bar{\varphi}\right) \mathrm{d} x=0, \quad \forall \varphi \in \widetilde{H}_{0}^{2}(\Omega) \\
& \int_{\partial \Omega} \bar{\varphi} \frac{k^{2} u}{\eta} \mathrm{d} s-\int_{\Omega} \frac{1}{n-1}\left(\Delta u+k^{2} u\right) \cdot\left(\Delta \bar{\varphi}+k^{2} \bar{\varphi}\right) \mathrm{d} x-\int_{\Omega} k^{2} u\left(\Delta \bar{\varphi}+k^{2} \bar{\varphi}\right) \mathrm{d} x=0 \\
& \int_{\partial \Omega} \bar{\varphi} \frac{k^{2} u}{\eta} \mathrm{d} s-\int_{\Omega} \frac{1}{n-1}\left(\Delta u+k^{2} u\right) \cdot\left(\Delta \bar{\varphi}+k^{2} \bar{\varphi}\right) \mathrm{d} x-k^{2}\left[-\int_{\Omega} \nabla u \cdot \nabla \bar{\varphi} \mathrm{d} x+\int_{\partial \Omega} u \frac{\partial \varphi}{\partial \nu} \mathrm{d} s\right]-k^{4} \int_{\Omega} u \cdot \bar{\varphi} \mathrm{d} x=0
\end{aligned}
$$

Since $\varphi \in \tilde{H}_{0}^{2}(\Omega)$, then $\int_{\partial \Omega} u(\partial \varphi / \partial \nu) \mathrm{d} s=0$; so, the following identity is

$$
\int_{\partial \Omega} \bar{\varphi} \frac{k^{2} u}{\eta} \mathrm{d} s-\int_{\Omega} \frac{1}{n-1}\left(\Delta u+k^{2} u\right) \cdot\left(\Delta \bar{\varphi}+k^{2} \bar{\varphi}\right) \mathrm{d} x+k^{2} \int_{\Omega} \nabla u \cdot \nabla \bar{\varphi} \mathrm{d} x-k^{4} \int_{\Omega} u \cdot \bar{\varphi} \mathrm{d} x=0
$$

Multiplying by -1 both sides, we have the final result

$$
-\int_{\partial \Omega} \bar{\varphi} \frac{k^{2} u}{\eta} \mathrm{d} s+\int_{\Omega} \frac{1}{n-1}\left(\Delta u+k^{2} u\right) \cdot\left(\Delta \bar{\varphi}+k^{2} \bar{\varphi}\right) \mathrm{d} x-k^{2} \int_{\Omega} \nabla u \cdot \nabla \bar{\varphi} \mathrm{d} x+k^{4} \int_{\Omega} u \cdot \bar{\varphi} \mathrm{d} x=0
$$


We define the following sesquilinear forms:

$$
\begin{aligned}
A_{k}(u, \varphi) & =\left(\frac{1}{n-1}\left(\Delta u+k^{2} u\right),\left(\Delta \varphi+k^{2} \varphi\right)\right)_{\Omega}+k^{4}(u, \varphi)_{\Omega}, \\
B(u, \varphi) & =(\nabla u, \nabla \varphi)_{\Omega}+\int_{\partial \Omega} \bar{\varphi} \frac{u}{\eta} \mathrm{d} s .
\end{aligned}
$$

So, if $n_{*}>1$, we can write our TEP as $A_{k}(u, \varphi)-k^{2} B(u, \varphi)=0$, for all $\varphi \in \widetilde{H}_{0}^{2}(\Omega)$.
Next, we need to prove that we are in the conditions of the theorem, which means that the operators $A_{k}$ are selfadjoint positive definite bounded linear operators on $\widetilde{H}_{0}^{2}(\Omega)$ and the operator $B$ is self-adjoint and nonnegative compact bounded on $\widetilde{H}_{0}^{2}(\Omega)$.

Let us first show that operator $A_{k}$ has the property required. Since $n_{*}>1$, then $1 /(n-1)>\beta>0$ almost everywhere in $\Omega$. The following inequalities show that $A_{k}$ is a coercive sesquilinear form on $\widetilde{H}_{0}^{2}(\Omega) \times \widetilde{H}_{0}^{2}(\Omega)$.

$$
A_{k}(u, u) \geq \beta\left\|\Delta u+k^{2} u\right\|_{L^{2}}^{2}+k^{4}\|u\|_{L^{2}}^{2} \geq \beta\|\Delta u\|_{L^{2}(\Omega)}^{2}-2 \beta\|\Delta u\|_{L^{2}(\Omega)}^{2} k^{2}\|u\|_{L^{2}(\Omega)}^{2}+(\beta+1) k^{4}\|u\|_{L^{2}(\Omega)}^{4} .
$$

If we take any $\epsilon$ between $\beta$ and $\beta+1$, then we have

$$
\begin{aligned}
& \varepsilon\left(k^{2}\|u\|_{L^{2}(\Omega)}^{2}-\frac{\beta}{\varepsilon}\|\Delta u\|_{L^{2}(\Omega)}^{2}\right)^{2}+\left(\beta-\frac{\beta^{2}}{\varepsilon}\right)\left(\|\Delta u\|_{L^{2}(\Omega)}^{2}\right)^{2} \\
& \quad+(\beta+1-\varepsilon) k^{4}\|u\|_{L^{2}(\Omega)}^{4},
\end{aligned}
$$

for $\beta<\varepsilon<\beta+1$. Since the first term of (31) is nonnegative, we obtain

$$
A_{k}(u, u) \geq\left(\beta-\frac{\beta^{2}}{\varepsilon}\right)\left(\|\Delta u\|_{L^{2}(\Omega)}^{2}\right)^{2}+(\beta+1-\varepsilon) k^{4}\|u\|_{L^{2}(\Omega)}^{4}
$$

Since for $u \in \widetilde{H}_{0}^{2}(\Omega),(\partial u / \partial \nu)=0$, then we can use Poincare inequality $[7,14]$ and have

$$
\|\nabla u\|_{L^{2}(\Omega)} \leq \frac{1}{\lambda_{1}}(\Omega)\|\Delta u\|_{L^{2}(\Omega)}^{2}
$$

The choice of $\varepsilon$ guaranty now is

$$
A_{k}(u, u) \geq c_{1}\|\Delta u\|_{L^{2}(\Omega)}^{2}+c_{2} k^{4}\|u\|_{L^{2}(\Omega)}^{2}=C_{k}\|u\|_{H^{2}(\Omega)}^{2} .
$$

From Riesz representation theorem, we define the bounded linear operators

$$
A_{k}: \widetilde{H}_{0}^{2}(\Omega) \times \widetilde{H}_{0}^{2}(\Omega), \quad \text { by }\left(A_{k^{2}} u, v\right)_{H^{2}(\Omega)}=\mathscr{A}(u, v) \text {. }
$$

Since $n$ and $\beta$ are real the sesquilinear forms $A_{k}$ are Hermitian; so, the operators are self-adjoint.

Also, we see that $B(u, \varphi)=(\nabla u, \nabla \varphi)_{\Omega}+\int_{\partial \Omega} \bar{\varphi}(u / \eta) \mathrm{d} s$ is nonnegative because

$$
\begin{aligned}
B(u, u) & =(\nabla u, \nabla \bar{u})_{\Omega}+\int_{\partial \Omega} \bar{u} \frac{u}{\eta} \mathrm{d} s=\int_{\Omega} \nabla u \cdot \bar{u} \mathrm{~d} x+\int_{\partial \Omega} \bar{u} \frac{u}{\eta} \mathrm{d} s \\
& =\int_{\Omega}|\nabla u|^{2} \mathrm{~d} x+\int_{\partial \Omega} \frac{|u|^{2}}{\eta} \mathrm{d} s \geq 0 .
\end{aligned}
$$

Since from the assumption $\eta>0$.

In Theorem 1, we have shown that $\nabla u$ is in $H^{1}(\Omega)$ and $H^{1}(\Omega)$ is compactly embedded in $L^{2}(\Omega)$. Furthermore, $\int_{\partial \Omega}(1 / n) u \cdot \bar{\varphi}$ contains $L^{2}(\partial \Omega)$ norm of $u$ because $u$ is in $H^{3 / 2} \partial \Omega$ which is compactly embedded in $L^{2}(\partial \Omega)$; so, the operator

$$
B(u, \varphi): \widetilde{H}_{0}^{2}(\Omega) \times \widetilde{H}_{0}^{2}(\Omega), \quad \text { defined by }(B u, v)_{H^{2}(\Omega)}=\mathscr{B}(u, v),
$$

is a nonnegative compact self-adjoint operator. According to the theorem, we have to show that the operator $A_{k}-k^{2} B$ is positive for some $k_{0}$ on $\tilde{H}_{0}^{2}(\Omega)$. We will consider as above the case when $n>1$. From assumption $\eta>0$, we have the coersivity of operator $B$.

$$
\begin{aligned}
A_{k}(u, u)-k^{2} B(u, u) & =\left.\int_{\Omega} \frac{1}{n-1}\left(\Delta u+k^{2} u\right)\right|^{2} \mathrm{~d} x+k^{2} \int_{\Omega}|u|^{2} \mathrm{~d} x-k^{2}\left(\int_{\Omega}|\nabla u|^{2} \mathrm{~d} x+\int_{\partial \Omega}|u|^{2} \frac{1}{\eta} \mathrm{d} s\right) \\
& \geq \int_{\Omega} \frac{1}{n-1}\left|\left(\Delta u+k^{2} u\right)\right|^{2} \mathrm{~d} x+k^{2} \int_{\Omega}|u|^{2} \mathrm{~d} x-k^{2} \min \left(1, \frac{1}{\eta_{\min }}\right)\left(\int_{\Omega}|\nabla|^{2} \mathrm{~d} x+\int_{\partial \Omega}|u|^{2} \mathrm{~d} s\right) \\
& \geq C_{1}\|\Delta u\|_{L^{2}(\Omega)}^{2}+C_{2} k^{4}\|u\|_{L^{2}(\Omega)}^{2}-k^{2} \alpha \frac{1}{\lambda_{1}(\Omega)}\|\Delta u\|_{L^{2}(\Omega)}^{2} .
\end{aligned}
$$


Since $C_{1}$ does not depend on $k$, for $k$ small enough, there exists a $k_{0}$ such that the quantity $C_{1}-k^{2} \alpha\left(1 / \lambda_{1}(\Omega)\right)>0$. We proved that condition (1) of Theorem 2 is satisfied.

Next, we must show that assumption (2) of Theorem 2 is also satisfied. We prove this in Theorem 3.

Theorem 3. Assume that $n>1$ a.e in $\Omega$, then there exists infinitely many transmission eigenvalues for problem (1)-(4).

Proof. The well-known result for the spherically stratified domain given by Colton et al. $[15,16]$ is applied to arrive at the existence of T.E. First, let $S_{j}=S\left(x_{j}, \varepsilon\right)$ $:=x \in R^{m}, m=2,3:\left|x-x_{j}\right|<\varepsilon$, where $x_{j} \in \Omega$ and $\varepsilon>0$.

The number of balls $S_{j}$ such that $S_{i} \cap S_{j}=\Phi$ is denoted by $N(\varepsilon)$, where $\varepsilon$ is sufficiently small to have the inclusion $\overline{S_{j}} \subset \Omega$. For the following interior transmission problem, there exists infinitely many transmission eigenvalues [16].

$$
\begin{aligned}
\Delta w_{j}+k^{2} n_{*} w_{j} & =0, \quad \text { and } \Delta v_{j}+k^{2} v_{j}=0 \text { in } S_{j}, \\
w_{j}-v_{j} & =0, \quad \text { and } \frac{\partial w_{j}}{\partial v}-\frac{\partial v_{j}}{\partial \nu}=0, \text { on } \partial S_{j},
\end{aligned}
$$

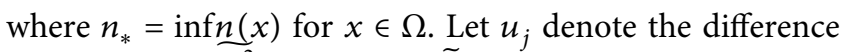
$u_{j}=w_{j}-v_{j} \in \widetilde{H_{0}^{2}}\left(S_{j}\right)$ and let $\tilde{u}_{j}$ be the extension of $u_{j}$ by zero to $\Omega$ and $H_{0}^{2}(\Omega)=\left\{u \in H^{2} \quad(\Omega)\right.$, such that $u=0$, in $\Omega,(\partial u / \partial \nu)=0$ on $\partial \Omega\}$. Obviously, $H_{0}^{2}(\Omega) \subset \tilde{H}_{0}^{2}(\Omega)$, so $\tilde{u}_{j} \in \widetilde{H_{0}^{2}}(\Omega) . \tilde{u}_{j}$ is orthogonal to $\tilde{u}_{i}$ for all $j \neq i$ in $\widetilde{H_{0}^{2}}(\Omega)$ since their support is disjoint. Let denote $\widetilde{H}_{N(\varepsilon)}=$ span $\left(\tilde{u}_{1}, \tilde{u}_{2}, \ldots, \tilde{u}_{N \varepsilon}\right) . \tilde{H}_{N(\varepsilon)}$ forms an $N(\varepsilon)$-dimensional subspace of $H_{0}^{2}(\Omega)$. Let $k=k_{j}$ be an eigenvalue of (39) and (40) and $u_{j}=w_{j}-v_{j}$ the corresponding eigenfunctions, then

$$
\begin{aligned}
0= & \int_{\Omega} \frac{1}{n_{*}-1}\left(\Delta \tilde{u}_{j}+k^{2} \tilde{u}_{j}\right) \cdot\left(\Delta \overline{\tilde{u}_{j}}+k^{2} \overline{\tilde{u}_{j}}\right) \mathrm{d} x \\
\leq & \int_{S_{j}} \frac{1}{n_{*}-1}\left(\Delta \tilde{u}_{j}+k^{2} \tilde{u}_{j}\right) \cdot\left(\Delta \overline{\tilde{u}_{j}}+k^{2} \tilde{\tilde{u}_{j}}\right) \mathrm{d} x \\
= & \int_{S_{j}} \frac{1}{n_{m}-1}\left(\left|\Delta \tilde{u}_{j}+k^{2} \tilde{u}_{j}\right|^{2}\right) \mathrm{d} x \\
& +k^{4} \int_{S_{j}}\left|\tilde{u}_{j}\right|^{2} \mathrm{~d} x-k^{2} \int_{S_{j}}\left|\nabla \tilde{u}_{j}\right|^{2} \mathrm{~d} x .
\end{aligned}
$$

We denote by $k_{\varepsilon}$ the first transmission eigenvalue, which is the same for each of these balls since they have the same radius and refractive index. This eigenvalue $k_{\varepsilon}$ has the respective eigen functions $\widetilde{w}_{j}, \widetilde{v}_{j}$, and $\widetilde{u}_{j}=\widetilde{w}_{j}-\widetilde{v}_{j}$. Then, we have

$$
\begin{array}{r}
\mathscr{A}_{k_{\varepsilon}}\left(\tilde{u}_{j}, \tilde{u}_{j}\right)-k_{\varepsilon}^{2} \mathscr{B}\left(\tilde{u}_{j}, \tilde{u}_{j}\right)=\int_{\Omega} \frac{1}{n_{m}-1} \\
\left(\left|\Delta \tilde{u}_{j}+k_{\varepsilon}^{2} \tilde{u}_{j}\right|^{2}\right) \mathrm{d} x+k_{\varepsilon}^{4} \int_{\Omega}\left|\tilde{u}_{j}\right|^{2} \mathrm{~d} x-k_{\varepsilon}^{2} \int_{\Omega}\left|\nabla \tilde{u}_{j}\right|^{2} \mathrm{~d} x-k_{\varepsilon}^{2} \int_{\partial \Omega} \frac{1}{\eta}\left|\tilde{u}_{j}\right|^{2} \mathrm{~d} s \\
\leq \int_{S_{j}} \frac{1}{n_{m}-1}\left(\left|\Delta \tilde{u}_{j}+k^{2} \tilde{u}_{j}\right|^{2}\right) \mathrm{d} x+k^{4} \int_{S_{j}}\left|\tilde{u}_{j}\right|^{2} \mathrm{~d} x-k^{2} \int_{S_{j}}\left|\nabla \tilde{u}_{j}\right|^{2} \mathrm{~d} x=0 .
\end{array}
$$

So, $\mathscr{A}_{k_{\varepsilon}}(u, u)-k_{\varepsilon}^{2} \mathscr{B}(u, u) \leq 0$. We are in the conditions of Theorem 2 , so they are $N(\varepsilon)$ eigenvalues in the interval $\left(0, k_{\varepsilon}\right]$.

\section{Conclusions}

In this paper, we studied in detail the discreteness and the existence of transmission eigenvalues of the given transmission problem in inhomogeneous media. We first used the variational approach to the problem which allowed us to present the TEP as a quadratic form related to $k$ to prove that the spectrum of the transmission eigenvalue problem is discrete. From a practical point of view, the discreteness is needed to guarantee that the numerical methods such as linear sampling succeed in the reconstruction of the scatterer of an inhomogeneous medium. The most important part of the work is the proof of the existence of the transmission eigenvalues. For this, we used a very important theorem of [8]. The existence of the eigenvalues is very important because they provide information about material properties of the scattering media and can be determined from scattering data and since they provide information about material properties of the scattering object and the transmission eigenvalues can play an important role in a variety of problems in target identification.

\section{Data Availability}

All data required for this study are included within this article.

\section{Conflicts of Interest}

The authors declare that they have no conflicts of interest.

\section{References}

[1] F. Cakoni, D. Gintides, and D. Gintides, "New results on transmission eigenvalues," Inverse Problems \& Imaging, vol. 4, no. 1, pp. 39-48, 2010.

[2] F. Cakoni, D. Colton, and H. Haddar, Inverse Scattering Theory and Transmission Eigenvalues, Society for Industrial and Applied Mathematics, Philadelphia, PA, USA, 2016.

[3] F. Cakoni and D. Colton, Qualitative Methods in Inverse Scattering Theory, Springer, Berlin, Germany, 2006.

[4] F. Cakoni and H. Haddar, "Transmission eigenvalues," Inverse Problems, vol. 29, no. 10, p. 100201, 2013. 
[5] F. Cakoni, D. Colton, and H. Haddar, "The interior transmission problem for regions with cavities," SIAM Journal on Mathematical Analysis, vol. 42, no. 1, pp. 145-162, 2010.

[6] B. Cobani and L. Subashi, "On the well-posedness of a transmission eigenvalue problem," Advances in Mathematics: Scientific Journal, vol. 9, pp. 8997-9003, 2020.

[7] O. Bodarenko, I. Harris, and A. Kleefeld, "The interior transmission eigenvalue problem for an inhomogeneous media with a conductive boundary," Applicable Analysis, vol. 96, no. 1, pp. 2-22, 2017.

[8] F. Cakoni, D. Colton, and H. Haddar, Inverse Scattering Theory and Transmission Eigenvalues, Vol. 88, SIAM Publications, Philadelphia, PA, USA, 2016.

[9] A. Bressan, Lecture Notes on Functional Analysis with Applications to Linear Partial Differential Equations, American Mathematical Society, Providence, RI, USA, 2013.

[10] F. Sayas, T. Brown, and M. Hassell, Variational Techniques for Elliptic Partial Differential Equations, Taylor and Francis Group, Abingdon, UK, 2019.

[11] L. C. Evans, Partial Differential Equations, American Mathematical Society, Providence, RI, USA, Second edition, 2010.

[12] S. Salsa, Partial Differential Equations in Action: From Modelling to Theory, Springer, Berlin, Germany, 2010.

[13] F. Cakoni, D. Colton, and D. Gintides, "The interior transmission eigenvalue problem," SIAM Journal on Mathematical Analysis, vol. 42, no. 6, pp. 2912-2921, 2010.

[14] F. Cakoni, D. Gintides, and H. Haddar, "The existence of an infinite discrete set of transmission eigenvalues," SIAM Journal on Mathematical Analysis, vol. 42, no. 1, pp. 237-255, 2010.

[15] D. Colton, A. Kirsch, and L. Päivärinta, "Far-field patterns for acoustic waves in an inhomogeneous medium," SIAM Journal on Mathematical Analysis, vol. 20, no. 6, pp. 1472-1483, 1989.

[16] D. Colton, L. Päivärinta, and J. Sylvester, "The interior transmission problem," Inverse Problems and Imaging, vol. 1, no. 1 , pp. $13-28,2007$. 